\title{
用较少变量函数的叠合逼近多变量函数 *
}

近年来, 用一个固定函数的复合来逼近多 元连续函数问题引起了工程师及数学家的广 泛的兴趣, 因为它是神经网络及小波分析中的 一个根本问题，在以往的一系列文章中 ${ }^{[1-5]}$ 我们讨论了一个一元函数的复合对多元函数 的逼近. 本文将讨论一个 $s$ 元函数的复合对 $n$ 元函数的逼近 $(s \leqslant n)$.

为了叙述方便, 文中所出现的所有 $\lambda_{i} \neq$ $0, K$ 为 $\mathbb{R}^{n}$ 中的一个紧集, $C(K)$ 为 $K$ 上连续 函数全体, $\mathrm{L}^{p}(K)$ 为 $K$ 上 $\mathrm{L}^{p}$ 可积函数全体, $S\left(\mathbb{R}^{n}\right)$ 为 $\mathbb{R}^{n}$ 上 Schwartz 函数全体.

首先, 设 $s=n$, 有

定理 1 假设 $G\left(x_{1}, \cdots, x_{n}\right) \in C\left(\mathbb{R}^{n}\right) \cap$ $S^{\prime}\left(\mathbb{R}^{n}\right)$, 即 $G \in C\left(\mathbb{R}^{n}\right)$ 且积分 $\int_{\mathbb{R}}^{-} G(x) s(x) \mathrm{d} x$ 对任一 $s \in S\left(\mathbb{R}^{n}\right)$ 有意义. 则下述函数 $\sum_{i=1}^{n} c_{i} G\left(\frac{x_{1}-x_{1}^{i}}{\lambda_{i 1}}, \cdots, \frac{i_{n}-x_{n}^{i}}{\lambda_{i n}}\right)$ 全体在 $C(K)$ 中稠密的充要条件是 $G(x)$ 不为下述形式 函数

$$
\begin{gathered}
G\left(x_{1}, \cdots, x_{n}\right)= \\
\sum_{i=1}^{n} \sum_{j=1}^{m_{i}} c_{i j} x_{i}^{j} G_{i j}\left(x_{1}, \cdots, x_{i-1}, x_{i+1}, \cdots, x_{n}\right),
\end{gathered}
$$

其中 $G_{i j} \in C\left(\mathbb{R}^{n-1}\right) \cap S^{\prime}\left(\mathbb{R}^{n-1}\right)$.

定理 2 设 $G\left(x_{1}, \cdots, x_{n}\right) \in \mathrm{L}_{\mathrm{Loc}}\left(\mathbb{R}^{n}\right) \cap$ $S^{\prime}\left(\mathbb{R}^{n}\right)$, 则下述函数

$\sum_{i=1}^{n} c_{i} G\left(\frac{x_{1}-x_{1}^{i}}{\lambda_{i 1}}, \cdots, \frac{x_{n}-x_{n}^{i}}{\lambda_{i n}}\right)$ 全体在 $\mathrm{L}_{p}(K)$ 中稠密的充要条件是 $G(x)$ 不为下述形式的 函数

$$
\begin{gathered}
G\left(x_{1}, \cdots, x_{n}\right)= \\
\sum_{i=1}^{n} \sum_{j=1}^{m_{i}} c_{i j} x_{i}^{j} G_{i j}\left(x_{1}, \cdots, x_{i-1}, x_{i+1}, \cdots, x_{n}\right),
\end{gathered}
$$

其中 $G_{i j} \in \mathrm{L}_{\mathrm{Loc}}\left(\mathbb{R}^{n-1}\right) \cap S^{\prime}\left(\mathbb{R}^{n-1}\right)$.

定理 3 设 $G\left(x_{1}, \cdots, x_{n}\right) \in C\left(\mathbb{R}^{n}\right) \cap$ $S^{\prime}\left(\mathbb{R}^{n}\right)$, 若 $\sum_{i=1}^{n} c_{i} G\left(\frac{x_{1}-x_{1}^{i}}{\lambda_{i 1}}, \cdots, \frac{x_{n}-x_{n}^{i}}{\lambda_{i n}}\right)$ 全 体在 $C(K)$ 中不稠密, 则存在一个以原点为项 点的雉 $C$, 使 $G$ 的 Fourier 变换 $G(\hat{V})=0$ 对 $V \in C$ 成立.

定理 4 设非零函数 $G\left(x_{1}, \cdots, x_{n}\right) \in$ $\mathrm{L}^{2}\left(\mathbb{R}^{n}\right)$, 则 $\sum_{i=1}^{n} c_{i} G\left(\frac{x_{1}-x_{1}^{i}}{\lambda_{i 1}}, \cdots, \frac{x_{n}-x_{n}^{i}}{\lambda_{i n}}\right)$ 全 体在 $\mathrm{L}^{2}\left(\mathbb{R}^{n}\right)$ 中稠密.

下面讨论 $(s \leqslant n)$ 情形, 设 $F: \mathbb{R}^{s} \rightarrow \mathbb{R}$ 满足 $F \in C\left(\mathbb{R}^{s}\right) \cap S^{\prime}\left(\mathbb{R}^{s}\right) . A=\left(a_{i j}\right)_{i=1, j=1}^{s, n}$ 为一个 $s \times n$ 矩阵, 它满足: 对任意 $j$, 至少有一个 $i$, 使 $a_{i j} \neq 0, D$ 为所有对角阵 $D=\operatorname{diag}\left[d_{1}, \cdots\right.$, $d_{n}$ ]全体, 其中所有 $d_{i} \neq 0$, 我们将给出列函数

$$
\mathscr{A}_{F}=\left\{F(A D X-t): D \in \mathscr{D}, t \in \mathbb{R}^{s}\right\}
$$

全体在 $C(K)$ 中稠密的条件.

显然, 当 $s=1$ 时,这个问题就是以前我们 讨论过的 $\mathrm{ABF}$ 神经网络的逼近问题.

上述一般形式的逼近问题包含了下述逼 近问题, 即下述形式函数

$$
U_{F}=\{F(A X-t): A \in \mathscr{A}, t \in \mathbb{R}\}
$$

在 $C(K)$ 中的稠密性问题. 其中 $\mathscr{A}$ 遍历所有 $s \times n$ 矩阵 $(s \leqslant n)$. 这类逼近问题已经被一 些学者研究过.

* 国家教委博士点基金及上海市科学基金资助项目 
定理 5 设 $F\left(u_{1}, \cdots, u_{s}\right) \in C\left(\mathbb{R}^{s}\right) \cap$ $S^{\prime}\left(\mathbb{R}^{s}\right)$, 即 $F \in C\left(\mathbb{R}^{s}\right)$ 且 积 分 $\int_{\mathbb{R}^{s}} F(u) s(u) \mathrm{d} u$ 对任一 $S \in S\left(\mathbb{R}^{s}\right)$ 有意义, $D, \mathscr{D}$ 定义如上, 则存在一个 $A$ 使下列函数集 $\mathscr{A}_{F}=\left\{F(A D X-t): D \in \mathscr{D}, t \in \mathbb{R}^{s}\right\}$ 在 $C(K)$ 中稠密的充要条件为 $F$ 不是一个多 项式.

类似地, 我们有

定理 6 设 $F\left(x_{1}, \cdots, x_{n}\right) \in \mathrm{L}_{\mathrm{Loc}}^{P}\left(\mathbb{R}^{s}\right) \cap$ $S^{\prime}\left(\mathbb{R}^{s}\right), A, D, \mathscr{D}$ 定义如前. 则下述函数全体

$$
\mathscr{A}_{F}=\left\{F(A D X-t): D \in \mathscr{D}, t \in \mathbb{R}^{d}\right\}
$$

在 $\mathrm{L}^{p}(K)$ 中稠密的充要条件为 $F$ 不是一个多 项式.

\section{参考文献}

1 Chen Tianping, Chen Hong. Approximation to continuous functionals by neural networks with application to dynami- cal systems. IEEE Trans on Neural Networks, 1993, 4 (6) : $910 \sim 918$

2 Chen Tianping. Approximation problems in system identification with neural networks. Science in China, Ser A, 1994, 37(4) : 414 421

3 Chen Tianping, Chen Hong, Liu Rueywen. Approximation capability in $C\left(\overline{\mathrm{R}}^{\mathrm{n}}\right)$ by multilayer feedforward networks and related problems. IEEE Trans on Neural Networks, 1995, 6(1):25 30

4 Chen Tianping, Chen Hong. Approximation capability to functions of several variables, nonlinear functionals and operators by radial basis function neural networks. IEEE Trans on Neural Networks, 1995, 6(4): 904 910

5 Chen Tianping, Chen Hong. Universal approximation to nonlinear operators by neural networks with arbitrary activation functions and its application to dynamic systems. IEEE Trans on Neural Networks, 1995, 6(4):911 917

\section{陈天平}

(复旦大学数学系, 上海 200433)

陈 弘

(Sun Microsystems, USA)

\section{Shimizu-Leutbecher 不等式及 Jorgensen 不等式 在高维 Möbius 群中的推广}

众所周知, Jorgensen 不等式是研究平面 Möbius 群的有力工具. 由于高维 Möbius 群 与平面情形存在着本质差异, 如何在高维 Möbius 群中建立相应的 Jorgensen 不等式显 得非常重要, 已有许多作者对此十分活跃的课 题进行了研究, 见文献[1 3].

本文利用高维 Möbius 变换的 Clifford 矩 阵表示, 讨论了高维空间中不同条件下的 ShimizutLeutbecher 不等式和 Jorgensen 不等 式. 并推广了文献 $[2,3]$ 中的一些主要结果 (定理 3,4).

记 $M\left(\overline{\mathbb{R}}^{n}\right)$ 为所有作用在 $\overline{\mathbb{R}}^{n}$ 上的保向 Möbius 变换组成的群. 对任意 $f \in M\left(\overline{\mathbb{R}}^{n}\right)$,
存在唯一的 Clifford 矩阵 $\left(\begin{array}{ll}a & b \\ c & d\end{array}\right) \in P S L(2$, $\left.\Gamma_{n}\right) /\{ \pm I\}$ 满足

$$
f(x)=(a x+b)(c x+d)^{-1},
$$

其中 $x \in \overline{\mathbb{R}}^{n}$, 见文献 $[1,2]$. 于是我们等同 $f$ 与 $\left(\begin{array}{ll}a & b \\ c & d\end{array}\right)$, 记为 $f=\left(\begin{array}{ll}a & b \\ c & d\end{array}\right)$.

称 $f$ 是严格抛物的, 若 $f$ 共轭于 $\left(\begin{array}{ll}1 & t \\ 0 & 1\end{array}\right)(t>0) ; f$ 是双曲的, 若 $f$ 共轭于 $\left(\begin{array}{ll}r & 0 \\ 0 & r^{-1}\end{array}\right)(r>0, r \neq 1) . f$ 是向量型的, 若 $b^{*}=b, c^{*}=c$ 且 $\operatorname{tr}(f)=a+d^{*} \in \mathbb{R}^{1}$. 